СПкЯ в репродуктивном возрасте: фенотипическое разнообразие и диагностические подходы (обзор литературы)

\author{
Л.М. Лазарева, Э.М. Шарифулин, Л.В. Беленькая, Л.В. Сутурина \\ ФГБНУ «Научный центр проблем здоровья семьи и репродукции человека» Министерства науки и высшего образования Российской \\ Федерации; Россия, г. Иркутск
}

\title{
PEЗЮME
}

\begin{abstract}
Цель обзора: систематизировать современные сведения об эпидемиологии, патофизиологии и диагностике синдрома поликистозных яичников (СПКЯ) и его фенотипов, а также об отдаленных последствиях заболевания у женщин репродуктивного возраста.

Основные положения. Для СПКЯ характерны гиперандрогенизм, овуляторная дисфункция и поликистозная структура яичников, выявляемая при УзИ. Ключевая особенность синдрома - избыточная продукция андрогенов яичниками. У пациенток с СПКЯ повышен риск кардиометаболических и онкологических заболеваний, в том числе рака эндометрия.

Заключение. Для диагностики СПКЯ необходимо оценивать менструальную функцию и проявления андрогензависимой дермопатии, проводить УЗИ для оценки структуры яичников и гормональное тестирование для выявления гиперандрогенемии. Обязательный компонент диагностического алгоритма - исключение заболеваний со сходными проявлениями.

Ключевые слова: синдром поликистозных яичников, синдром Штейна - Левенталя, инсулинорезистентность, репродуктивный возраст, гирсутизм, олиго-/ановуляция, фенотипы, гиперандрогения.
\end{abstract}

Вклад авторов: Лазарева Л.М. - выбор тематики обзора, поиск литературных источников, написание текста; Шарифулин Э.М., Беленькая Л.В. - поиск литературных источников; Сутурина Л.В. - определение цели и задач обзора, научное редактирование, утверждение рукописи для публикации.

Конфликт интересов: авторы заявляют об отсутствии возможных конфликтов интересов.

Для цитирования: Лазарева Л.М., Шарифулин Э.М., Беленькая Л.В., Сутурина Л.В. СПКЯ в репродуктивном возрасте: фенотипическое разнообразие и диагностические подходы (обзор литературы). Доктор.Ру. 2020; 19(6): 50-56. DOI: 10.31550/1727-2378-2020-19-6-50-56

\section{Polycystic Ovary Syndrome in Women of Reproductive Age: Phenotypic Variety and Diagnostic Approaches. Review of Literature}

\author{
L.M. Lazareva, E.M. Sharifulin, L.V. Belenkaya, L.V. Suturina \\ Scientific Center for Family Health and Human Reproduction (a Federal Government-funded Scientific Institution), Russian Federation \\ Ministry of Science and Higher Education; 16 Timiryazev St., Irkutsk, Russian Federation 664003
}

\section{ABSTRACT}

Objective of the Review: To systematize current information of the epidemiology, pathophysiology, and diagnosis of polycystic ovary syndrome (PCOS) and its phenotypes as well as its long-term sequelae in women of reproductive age.

Key Points: Typical manifestations of PCOS include hyperandrogenism, ovarian dysfunction, and a polycystic ovarian pattern identified by ultrasound. The key feature of this syndrome is ovarian overproduction of androgens. Patients with PCOS are at higher risk for cardiometabolic disorders and neoplasms, including endometrial cancer.

Conclusion: The diagnosis of PCOS requires assessment of the menstrual cycle and manifestations of androgen-dependent skin disorders, ultrasound evaluation of the ovarian structure, and hormonal testing for hyperandrogenemia. Ruling out disorders with similar manifestations is an essential component of the diagnostic process.

Keywords: polycystic ovary syndrome, Stein-Leventhal syndrome, insulin resistance, reproductive age, hirsutism, oligoovulation, anovulation, phenotypes, hyperandrogenism.

Contributions: Dr. L.M. Lazareva chose the subject of the review, searched for publications, and wrote the paper. Dr. E.M. Sharifulin and Dr. L.V. Belenkaya searched for publications. Dr. L.V. Suturina outlined the goal and objectives of the review, provided scientific editing, and approved the final version of the manuscript submitted for publication.

Conflict of interest: The authors declare that they do not have any conflict of interests.

For citation: Lazareva L.M., Sharifulin E.M., Belenkaya L.V., Suturina L.V. Polycystic Ovary Syndrome in Women of Reproductive Age: Phenotypic Variety and Diagnostic Approaches. Review of Literature. Doctor.Ru. 2020; 19(6): 50-56. (in Russian) D0I: 10.31550/1727-2378-2020-19-6-50-56

Лазарева Людмила Михайловна (автор для переписки) - к. м. н., научный сотрудник лаборатории гинекологической эндокринологии ФГБНУ НЦ ПЗСРЧ Минобрнауки России. 664003, Россия, г. Иркутск, ул. Тимирязева, д. 16. ORCID: https://orcid.org/0000-0002-7662-8529. E-mail: lirken_@mail.ru

Шарифулин Эльдар Махарамович - аспирант, врач - акушер-гинеколог отдела охраны репродуктивного здоровья ФГБНУ НЦ ПЗСРч Минобрнауки России. 664003, Россия, г. Иркутск, ул. Тимирязева, д. 16. ORCID: https://orcid.org/0000-0002-7245-9289. E-mail: sharifulja@mail.ru (Окончание на с. 51.) 
C индром поликистозных яичников (СПКЯ) признан наиболее часто встречающейся эндокринопатией среди женщин репродуктивного возраста [1-6]. Распространенность СПКЯ существенно варьирует и неодинакова в различных популяциях. В частности, она различается в городских и сельских поселениях [7], а также зависит от расовой и этнической принадлежности $[8,9]$. Среди женщин, обращающихся в медицинские учреждения, частота СПКЯ значительно выше популяционной и составляет от $46 \%$ по критериям диагностики Национальных институтов здоровья США (англ. National Institutes of Health, NIH) до 73,3\% по Роттердамским критериям диагностики (описание критериев см. ниже. - Прим. $a в m$.). В неселективной с медицинской точки зрения популяции женщин репродуктивного возраста распространенность СПкЯ по вышеназванным критериям колеблется от 4-6,6\% до $6-21 \%$ соответственно $[1,5,8,10]$.

СПКЯ встречается у каждой четвертой пациентки с ожирением [11], а при клинических проявлениях гиперандрогенизма его частота достигает 72,6\% [12].

СПКЯ характеризуется гиперандрогенией (ГА) и сложным спектром репродуктивных расстройств, таких как менструальная дисфункция, невынашивание беременности, бесплодие [13], а также ассоциирован с повышенным риском метаболических нарушений, в том числе инсулинорезистентности и сахарного диабета 2 типа [14]. Кроме того, существует связь между СПКЯ и риском развития карциномы эндометрия, сердечно-сосудистых и церебральных катастроф [15], депрессии и психосексуальных расстройств [16], апноэ сна $[3,17]$. Беременность у женщин с СпКЯ сопряжена с повышенным риском развития акушерских и перинатальных осложнений, включая гестационный диабет с макросомией плода [17]. Имеются сведения о том, что сПКЯ ассоциирован с более высокой частотой госпитализаций, не связанных с травмами и акушерскими факторами [3].

Первые описания СПКЯ опубликованы много лет назад [18, 19], тем не менее этиология и патофизиология этого расстройства продолжают активно изучаться. Доказана многофакторность СПКЯ [20], подчеркнуто значение генетических и эпигенетических факторов в его развитии [21].

К основным механизмам патогенеза СПКЯ относят нарушения стероидпродуцирующей функции яичников [22] с увеличением продукции андрогенов и нарушением их преобразования в эстрогены, а также повышение уровня свободного тестостерона на фоне низкого содержания глобулина, связывающего половые гормоны (ГСПГ) [23]. В свою очередь, гиперандрогенизм ассоциирован с абдоминальным типом ожирения и изменениями липидного профиля [24]. Кроме того, СПКЯ характеризуется наличием гонадотропной дисфункции, что закономерно приводит к ановуляции или как минимум к недостаточности прогестерона [22]. Наряду с этим ановуляция и мелкокистозная трансформация яичников являются следствием так называемого фолликулярного ареста, ассоциированного с чрезмерной секрецией антимюллерового гормона (АМГ). 50-90\% женщин с СПкЯ имеют гиперинсулинемию и инсулинорезистентность [14], которые усугубляют гиперандрогенизм, ожирение и овариальную дисфункцию [25].

Гиперандрогенизм и висцеральный тип ожирения, свойственные пациенткам с СПКЯ, сопровождаются дисбалансом между провоспалительными медиаторами, противовоспалительнымим цитокинами и, как следствие, хроническим системным воспалением и окислительным стрессом [26, 27]. Причем степень проявления системного воспалительного ответа прямо пропорциональна степени ожирения и ИМТ [28], тогда как выраженность окислительного стресса при СПКЯ от ИМТ не зависит [26]. Однако в клинической практике определение маркеров хронического воспаления и оксидативного стресса пока не распространено.

Исторически диагностика СПКЯ основана на критериях, утвержденных и представленных в различных консенсусах. Первые согласованные подходы к диагностике спкя были предложены в апреле 1990 г. Национальным институтом здоровья детей и развития человека им. Юнис Кеннеди Шрайвер (англ. Eunice Kennedy Shriver National Institute of Child Health and Human Development, NICHD), входящим в состав NIH [29]. В 2003 г. в Роттердаме экспертами Европейского общества репродукции человека и эмбриологии (англ. European Society of Human Reproduction and Embryology) и Американского общества репродуктивной медицины (англ. American Society of Reproductive Medicine, ASRM) были приняты новые критерии диагностики СПКЯ [30]. В 2006 г. свою версию критериев предложили эксперты Общества по гиперандрогениям и СПКЯ (англ. Androgen Excess and PCOS Society, AE-PCOS) [31]. Последнее руководство, посвященное диагностике СПКЯ и ведению пациенток с этим синдромом, было опубликовано в 2018 г. [3].

Согласно критериям NIH (1990), необходимым условием для диагностики СПкя являлось наличие олиго-/ановуляции и гиперандрогенизма (после исключения состояний с похожими симптомами) [29]. При этом обнаружение поликистозной структуры яичников по УзИ не рассматривалось как обязательный признак СПКя. После принятия Роттердамского консенсуса (2003) диагностически значимым стало считаться наличие как минимум двух признаков из трех: олиго-/ановуляции, поликистозной структуры яичников, клинического и/или биохимического гиперандрогенизма. Введение данных критериев закономерно привело к увеличению частоты выявления СПКЯ за счет неандрогенного и овуляторного фенотипов. Критерии СПКЯ, принятые AE-PCOS (2006), концентрировались на проявлениях гиперандрогенизма, который был признан необходимым признаком СПКЯ. По мнению экспертов AE-PCOS, СПКЯ следовало диагностировать при обязательном наличии гиперандрогенизма в сочетании с менструальной и овуляторной дисфункцией и/или поликистозной структурой яичников по данным УзИ [32]. В результате последующих обсуждений было принято решение рекомендовать для диагностики СПкя Роттердамский подход, охватывающий критерии как NIH (1990), так и AE-PCOS (2006), с обязательной идентификацией фенотипа СПКЯ [33].

Беленькая Лилия Васильевна - к. м. н., старший научный сотрудник лаборатории физиологии и патологии эндокринной системы ФГБНУ НЦ ПЗСРЧ Минобрнауки России. 664003, Россия, г. Иркутск, ул. Тимирязева, д. 16. ORCID: https://orcid.org/0000-0003-4904-3709. E-mail:drblv@mail.ru

Сутурина Лариса Викторовна - д. м. н., профессор, главный научный сотрудник, руководитель отдела охраны репродуктивного здоровья ФГБНУ НЦ ПЗСРЧ Минобрнауки России. 664003, Россия, г. Иркутск, ул. Тимирязева, д. 16. ORCID: https://orcid.org/0000-0002-6271-7803. E-mail: Isuturina@mail.ru

(Окончание. Начало см. на с. 50.) 
Предложена следующая классификация фенотипов СПкя:

- фенотип А - все признаки СПКЯ (клинический гиперандрогенизм и/или гиперандрогенемия, овуляторная дисфункция и поликистозные яичники по данным ультрасонографии);

- фенотип В - ГА и олиго-/ановуляция;

- фенотип С - ГА и поликистозная структура яичников по данным УЗИ;

- фенотип D - олиго-/ановуляция и поликистозная структура яичников по данным ультрасонографии [33].

Фенотип А (классический) считается наиболее тяжелым: он характеризуется всеми тремя Роттердамскими критериями. Ожирение и инсулинорезистентность, высокий уровень АМГ, хотя и не входят в диагностические критерии, тесно связаны с этим фенотипом [33].

Фенотип В (также классический (NIH) фенотип) отличается выраженной менструальной дисфункцией и характеризуется более высокой частотой инсулинорезистентности и метаболических нарушений по сравнению с негиперандрогенными формами СПКЯ $[3,33]$.

При фенотипе C (овуляторном) пациентки имеют повышенные уровни андрогенов и липидов в сыворотке крови [33], а также умеренную гиперинсулинемию с более низкой частотой метаболического синдрома и гирсутизма, чем при прочих фенотипах СПКЯ.

У пациенток с фенотипом D (неандрогенным) в нескольких исследованиях показаны максимальное количество регулярных менструальных циклов, наиболее низкая степень метаболических нарушений $[3,23]$. У этих женщин регистрируются более низкие значения тестостерона и соотношения ЛГ/ФСГ в сыворотке крови, но значительно более высокие уровни ГСПГ в сравнении с пациентками с классическими фенотипами СПКЯ [34].

Некоторые данные свидетельствуют о том, что женщины с фенотипами СПКЯ А и В имеют более высокий риск стеатоза печени, чем пациентки с негиперандрогенным фенотипом заболевания и здоровые женщины. Самые высокие уровни АМГ также обнаружены в группах с классическими фенотипами СПКЯ [3].

В настоящее время диагностика синдрома основана на клинических и биохимических проявлениях ГА и овуляторной дисфункции, а также на результатах оценки ультразвуковой структуры яичников. Обновленные подходы к диагностике представлены в новом консенсусе (2018), который включает в себя рекомендации, базирующиеся на фактических данных, и затрагивает основные клинико-диагностические и лечебные аспекты СПкЯ [3].

Клиническими проявлениями гиперандрогенизма являются гирсутизм и (в меньшей степени) алопеция и акне. Термин «гирсутизм» означает чрезмерный рост терминальных волос у женщин и детей с мужским типом распределения. Для оценки гирсутизма в качестве показателя клинического гиперандрогенизма в настоящее время рекомендована стандартизированная модифицированная визуальная шкала Ферримана - Галлвея (D. Ferriman - J. Gallwey, mFG) [35]. При осмотре пациенток с подозрением на гирсутизм клиницисты должны оценивать наличие именно терминальных волос по мужскому типу оволосения на девяти различных участках тела (верхняя губа, подбородок, грудь, верхняя часть спины, нижняя часть спины, верхняя часть живота, нижняя часть живота, плечо, бедро) (рис. 1) [35, 36].

Сумма баллов по шкале $\mathrm{mFG} \geq 6$ указывает на гирсутизм [3]. Однако пороговое значение $\mathrm{mFG}$ при диагностике
Рис. 1. Схематическое представление

модифицированной шкалы Ферримана - Гал^вея.

Воспроизводится с разрешения авторов [36]

Fig. 1. Schematic representation of the modified

Ferriman-Gallwey scale. Reproduced by permission of the authors [36]
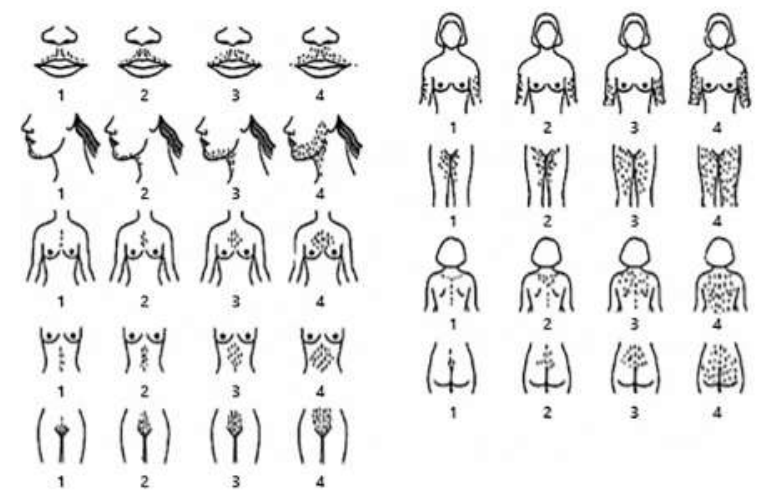

гирсутизма может различаться в зависимости, например, от расы (табл.).

Избыточный рост волос сам по себе не всегда является отражением ГА и может быть обусловлен семейными/этническими особенностями, отклонениями в выработке кортикостероидов и гормона роста, нарушением функции щитовидной железы, гиперпролактинемией или применением лекарственных препаратов. Гирсутизм необходимо отличать от гипертрихоза, который чаще характеризуется локализованным ростом мягких тонких волос (преимущественно на предплечьях или голенях), хотя возможен и их генерализованный избыточный рост. Иногда у пациентки наблюдается как гирсутизм, так и гипертрихоз. Гипертрихоз обычно не является следствием гиперандрогенизма, но гиперандрогенемия может усугубить его [36].

Шкала $\mathrm{mFG}$ имеет недостатки, связанные с субъективностью оценки состояния по ней как со стороны пациентки, так и, в меньшей степени, со стороны врача. При самооценке пациентке сложно оценить степень гирсутизма в труднодоступных для осмотра местах (например, в области ягодиц), тогда как врач может испытывать затруднения, оценивая выраженность гирсутизма, если пациенткой используются различные способы удаления волос. Таким образом, полезно проводить как самооценку [50], так и подсчет баллов опытным специалистом [51].

Для оценки алопеции принята стандартная визуальная шкала Людвига (рuс. 2) [3].

Наличие акне при диагностике ГА принимается во внимание только в случаях его сочетания с другими проявлениями заболевания, в частности с гирсутизмом.

Оценка биохимического гиперандрогенизма наиболее актуальна при постановке диагноза СПкЯ и/или определении фенотипа в тех случаях, когда клинические признаки ГА (в частности, гирсутизм) слабо выражены или отсутствуют, например в азиатских популяциях.

Характерной особенностью СПКЯ является избыток тестостерона. На современном этапе оптимальным методом оценки концентраций общего тестостерона считается жидкостная хроматография с масс-спектрометрией. Кроме того, для диагностики гиперандрогенемии рекомендуется определение содержания свободного/биодоступного тестостерона с применением расчетных методов. При нормальных концентрациях общего и свободного тестостерона дополнительными 
Таблица / Table

Критерии диагностики гирсутизма по шкаме Ферримана - ГамАвея в размичных попумяциях

Diagnostic criteria for hirsutism in various populations: the Ferriman-Gallwey score

\begin{tabular}{|c|c|c|c|}
\hline Авторы, год / Reference, year & Страна / Country & Paca / Race & $\begin{array}{c}\text { Минимальное порого- } \\
\text { вое значение шкалы / } \\
\text { Minimal threshold score }\end{array}$ \\
\hline $\begin{array}{l}\text { Diamanti-Kandarakis E. et al. } \\
(1999)^{*}[37]\end{array}$ & Греция / Greece & европеоидная / Caucasian & 6 \\
\hline Asunción M. et al. (2000) [38] & Испания / Spain & европеоидная / Caucasian & 8 \\
\hline Zargar A.H. et al. (2002) [39] & $\begin{array}{l}\text { Индия (Кашмир, Дардик) / } \\
\text { India (Kashmir, Dardik) }\end{array}$ & азиатская / Asian & 6 \\
\hline Sagsoz N. et al. (2004) [40] & Турция / Turkey & европеоидная / Caucasian & 8 \\
\hline Cheewadhanaraks S. et al. (2004) [41] & $\begin{array}{l}\text { Тайланд, Китай / Thailand, } \\
\text { China }\end{array}$ & азиатская / Asian & 3 \\
\hline DeUgarte C.M. et al. (2006) [42] & США / USA & $\begin{array}{l}\text { европеоидная, негроидная / } \\
\text { Caucasian, black }\end{array}$ & 8 \\
\hline Mehrabian F. et al. (2011) [43] & Иран / Iran & европеоидная / Caucasian & 8 \\
\hline Teede H. et al. (2010) [44] & Австралия Australia & европеоидная / Caucasian & 8 \\
\hline Zhao X. et al. (2011) [45] & Южный Китай / South China & азиатская / Asian & 5 \\
\hline Kim J.J. et al. (2011) [46] & Корея / Кorea & азиатская / Asian & 6 \\
\hline Repaci A. et al. (2011) [47] & Италия / Italy & европеоидная / Caucasian & 9 \\
\hline Escobar-Morreale H.F. et al. (2012) [48] & Испания / Spain & европеоидная / Caucasian & 10 \\
\hline Li R. et al. (2012) [49] & $\begin{array}{l}\text { Китай (10 провинций) / } \\
\text { China (10 provinces) }\end{array}$ & азиатская / Asian & $\begin{array}{l}4 \text { (усредненный балл / } \\
\text { mean score) }\end{array}$ \\
\hline
\end{tabular}

Примечание. Знаком (*) отмечена работа, основанная на Аанных оригинальной шкалы Ферримана Гал^вея, в остальных случаях применялась модифицированная версия шкалы (mFG).

Note: * Denotes a publication whose authors used the original Ferriman-Gallwey scale. All other authors used the modified Ferriman-Gallwey ( $\mathrm{mFG}$ ) scale.

Рис. 2. Стадии андрогенной алопеции по шкале

Аюдвига [3]

Fig. 2. Classification of androgenic alopecia by the Ludwig scale [3]

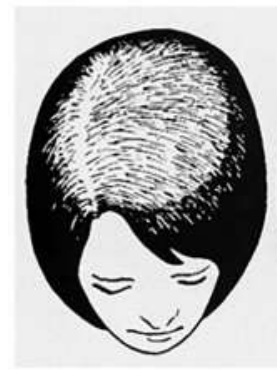

1

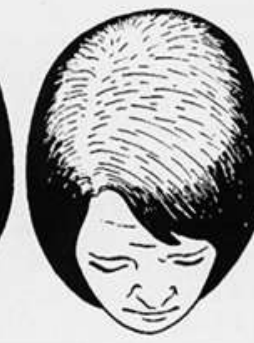

2

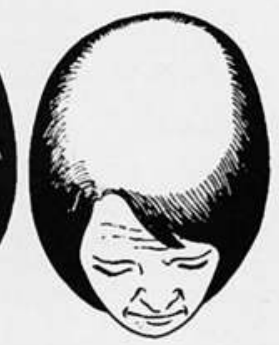

3 маркерами ГА при СПкЯ могут быть повышенные уровни андростендиона и дегидроэпиандростерона [3].

Диагностика олиго-/ановуляции, овариальной дисфункции основана на оценке менструальной и/или овуляторной функции. В репродуктивном возрасте нарушенным считается менструальный цикл продолжительностью менее 21 или более 35 дней, так как доказано, что это коррелирует с олиго-/ановуляцией. Диагностическое значение имеет также наличие в анамнезе менее 8 менструальных циклов в год. При нормальной длительности менструального цикла необходимо оценивать овуляторную функцию путем не менее чем двукратного измерения уровня прогестерона в лютеиновую фазу [3].
В клинической оценке структуры яичников у сексуально активных женщин стандартом является трансвагинальное УзИ. При использовании датчика частотой 8 МГц основаниями для диагностики поликистозных яичников считаются наличие 20 и более фолликулов хотя бы в одном яичнике, а также объем яичников, равный 10 мл и более, при присутствии доминантных фолликулов, кист или желтых тел. Подчеркнуто, что для диагностики СПКЯ объем яичников - значительно более надежный параметр, чем число фолликулов, особенно при использовании ультразвуковой аппаратуры, не обладающей современными характеристиками. У пациенток с ГА и нерегулярными менструальными циклами УзИ органов малого таза не является необходимым для постановки диагноза СПКя, однако имеет значение для уточнения клинического фенотипа заболевания. Кроме того, в консенсусе 2018 г. протокол УзИ органов малого таза рекомендован для повышения качества исследования [3].

Известно, что пациентки с СПкЯ имеют более высокие уровни АМГ, чем здоровые женщины [52], доказана корреляция концентрации АМГ с количеством антральных фолликулов вне зависимости от менструального цикла [52]. Появилась гипотеза о том, что АМГ может быть не просто маркером количества фолликулов, а эндокринным сигналом, играющим ключевую роль в патогенезе СПКЯ [53]. Замечено также, что уровень АМГ снижается с возрастом, при уменьшении клинических проявлений СПкЯ [54]. В связи с этим некоторые авторы рассматривают АМГ как биомаркер СПКЯ, и овариальной дисфункции в частности $[55,56]$. Однако данный маркер не используется в рутинной клинической 
практике из-за недостаточной надежности лабораторных тест-систем [57], изменчивости уровней гормона [54].

У пациенток с СПКЯ, независимо от степени ожирения, типа распределения жировой ткани и уровней андрогенов, часто выявляются резистентность к инсулину и гиперинсулинемия. Женщины с СПКЯ имеют высокий риск развития нарушения толерантности к глюкозе и сахарного диабета 2 типа [3]. В связи с вышеизложенным у всех женщин с СПкя должен оцениваться исходный гликемический статус [3]. Диагностика нарушений углеводного обмена основана на определении уровней глюкозы и гликированного гемоглобина в плазме крови натощак.

У женщин с СПКЯ группы высокого риска рекомендуется проведение перорального теста на толерантность к глюкозе [3]. К группе риска относятся женщины европеоидной расы с ИМТ более $25 \mathrm{kг} / \mathrm{m}^{2}$ и азиатской расы с ИМТ более $23 \mathrm{\kappa r} / \mathrm{M}^{2}$, пациентки с нарушениями углеводного обмена (гипергликемией натощак, нарушением толерантности к глюкозе или гестационным диабетом) в анамнезе, женщины с отягощенным семейным анамнезом по сахарному диабету и гипертонической болезни, а также представительницы этнических групп с высоким риском нарушений углеводного обмена [3]. Учитывая риск гестационного диабета, всем женщинам с СПКЯ следует предлагать тест на толерантность к глюкозе при планировании беременности или обращении по поводу бесплодия [3].

Появляется все больше подтверждений того, что обструктивное апноэ во сне (ОА) должно рассматриваться в качестве фактора риска кардиометаболических нарушений при СПкЯ. $0 A$ является относительно распространенным синдромом, характеризующимся хроническим нарушением сна в виде рецидивирующей полной (апноэ) или частичной (гипопноэ) обструкции верхних дыхательных путей во время сна. Стандартным диагностическим тестом в отношении ОА признана ночная полисомнография. Тяжесть ОА обычно оценивают по индексу апноэ-гипопноэ, который представляет собой общее количество эпизодов апноэ и гипопноэ за час сна, зафиксированное во время полисомнографии. ОА проявляется чрезмерной сонливостью в дневное время, когнитивной дисфункцией, снижением качества жизни и повышением риска несчастных случаев. Существуют также убедительные доказательства того, что ОА способствует развитию артериальной гипертензии, сердечно-сосудистых заболеваний, нарушений углеводного обмена. Доказано, что у взрослых пациенток с СПКЯ риск развития ОА в 9,74 раза выше, чем у женщин аналогичного возраста, не имеющих этого заболевания [58].

Чтобы своевременно выявить симптомы, связанные с OA (храп, ощущение утомления и усталости после сна, сонливость и разбитость в дневное время, признаки депрессии), рекомендуется проводить скрининг пациенток с СПКя, используя соответствующие опросники. Пациенткам, вошедшим в группу риска по ОА, рекомендовано дальнейшее обследование с помощью ночной полисомнографии [3].

Первые ссылки на связь рака эндометрия и СПкЯ были опубликованы в 1949 г., всего через 14 лет после первого классического описания СПкЯ Ф. Штейном и М. Левенталем [19]. В современных исследованиях также отмечена высокая частота встречаемости гистологически подтвержденных патологих состояний эндометрия, в том числе хронического эндометрита, у таких больных [59]. У пациенток с классическим (по критериям NIH) СПкЯ риск развития рака эндометрия в $\leq 6$ раз превышает популяционный [60], причем часто сохраняется до менопаузы. Вместе с тем в большинстве случаев опухоли являются высокодифференцированными и имеют благоприятный прогноз [61]. Наличие аномального эндометрия у пациенток, имеющих СПКЯ и нарушение в связи с этим процессов децидуализации и плацентации [62], сопровождается более частым развитием патологии беременности: невынашивания, преждевременных родов, преэклампсии и артериальной гипертензии беременных [63].

Для своевременного выявления патологических состояний эндометрия при СПкя проводится обследование с помощью трансвагинального УЗИ. Пациенткам с утолщенным эндометрием и/или имеющим факторы риска, например длительную аменорею, аномальные маточные кровотечения или лишний вес, рекомендуется биопсия эндометрия. Вместе с тем стандартизованных критериев и алгоритмов формирования групп риска для своевременного выявления патологических состояний эндометрия и прогнозирования клинических исходов у женщин с СПкЯ до настоящего времени не разработано. Поэтому предложено информировать пациенток с синдромом об имеющихся у них рисках [61].

Внимание исследователей традиционно привлекает связь спКЯ с депрессивными и тревожными симптомами. В различных странах и регионах проведено больше 30 исследований, в которых оценивались риски возникновения умеренных и тяжелых симптомов депрессии у женщин при наличии и отсутствии СПКЯ. В результате было отмечено, что у пациенток с СПКЯ вероятность возникновения депрессии выше, чем у женщин, не имеющих этого синдрома. Однако исследования имели недостатки, основным из которых стало то, что авторы сравнивали госпитальные группы с контролем из популяции [64]. Кроме того, остаются недостаточно изученными географические и расовые особенности распространенности депрессии у пациенток с СПкя.

Согласно последним рекомендациям (2018), следует выполнять скрининг тревожных и депрессивных симптомов у всех женщин при постановке диагноза СпкЯ [3]. В случае выявления эмоционального неблагополучия предложено привлекать квалифицированных специалистов и проводить психологическую терапию или фармакологическое лечение в соответствии с региональными практическими рекомендациями. Однако до настоящего времени не определен оптимальный интервал для осуществления скрининга тревожных и депрессивных симптомов у пациенток с СПКЯ. Кроме того, при скрининге необходимо учитывать сопровождающие СПкЯ факторы риска эмоциональных нарушений (такие как бесплодие, невынашивание беременности, ожирение, гирсутизм), а также принимать во внимание использование препаратов, способных провоцировать и усугублять депрессивные расстройства.

\section{ЗАКЛЮЧЕНИЕ}

Синдром поликистозных яичников (СПКЯ) представляет собой гетерогенное заболевание со сложным этиопатогенезом, включающим в себя генетические, эпигенетические, этнические компоненты, которые при взаимодействии друг с другом участвуют в формировании фенотипа и определяют отдаленные последствия синдрома.

Основными проявлениями СПКЯ являются гиперандрогенизм, овариальная и/или овуляторная дисфункция и поликистозные изменения яичников. У значительного числа пациенток с СпкЯ имеются инсулинорезистентность, гиперинсулинемия и повышенный риск развития метаболических осложнений, а также репродуктивных нарушений и патологических состояний эндометрия. Женщины с СПКЯ подвержены риску 
развития депрессивных состояний, которые коррелируют с избытком андрогенов и гиперинсулинизмом; депрессии характеризуются хроническим течением и обусловливают снижение качества жизни.

Таким образом, современные подходы к диагностике Спкя предусматривают оценку основных клинических проявлений заболевания, а также исключение состояний со сходной симптоматикой и обязательную верификацию клинического

\section{ЛИТЕРATУPA / REFERENCES}

1. Azziz R., Woods K.S., Reyna R., Key T.J., Knochenhauer E.S., Yildiz B.O. The prevalence and features of the polycystic ovary syndrome in an unselected population. J. Clin. Endocrinol. Metab. 2004; 89(6): 2745-9. DOI: 10.1210/jc.2003-032046

2. Yildiz B.0., Bozdag G., Yapici Z., Esinler I., Yarali H. Prevalence, phenotype and cardiometabolic risk of polycystic ovary syndrome under different diagnostic criteria. Hum. Reprod. 2012; 27(10): 3067-3. DOI: 10.1093/humrep/des232

3. Teede H.J., Misso M.L., Costello M.F., Dokras A., Laven J., Moran L. et al. Recommendations from the international evidence-based guideline for the assessment and management of polycystic ovary syndrome. Clin. Endocrinol. (Oxf.). 2018; 89(3): 251-68. D0I: 10.1111/cen.13795

4. Lizneva D., Suturina L., Walker W., Brakta S., Gavrilova-Jordan L., Azziz R. Criteria, prevalence, and phenotypes of polycystic ovary syndrome. Fertil. Steril. 2016; 106(1): 6-15. D0I: 10.1016/j. fertnstert.2016.05.003

5. Lizneva D., Kirubakaran R., Mykhalchenko K., Suturina L., Chernukha G., Diamond M.P. et al. Phenotypes and body mass in women with polycystic ovary syndrome identified in referral versus unselected populations: systematic review and meta-analysis. Fertil. Steril. 2016; 106(6): 1510-20.e2. DOI: 10.1016/j.fertnstert.2016.07.1121

6. Belenkaia L.V., Lazareva L.M., Walker W., Lizneva D.V., Suturina L.V. Criteria, phenotypes and prevalence of polycystic ovary syndrome. Minerva Ginecol. 2019; 71(3): 211-23. DOI: 10.23736/S00264784.19.04404-6

7. Deswal R., Nanda S., Ghalaut V.S., Roy P.S., Dang A.S. Cross-sectional study of the prevalence of polycystic ovary syndrome in rural and urban populations. Int. J. Gynaecol. Obstet. 2019; 146(3): 370-9. DOI: 10.1002/ijgo.12893

8. Fauser B.C., Tarlatzis B.C., Rebar R.W., Legro R.S., Balen A.H., Lobo R. et al. Consensus on women's health aspects of polycystic ovary syndrome (PCOS): the Amsterdam ESHRE/ASRM-Sponsored 3rd PCOS Consensus Workshop Group. Fertil. Steril. 2012; 97(1): 28-38.e25. DOI: 10.1016/j.fertnstert.2011.09.024

9. Wolf W.M., Wattick R.A., Kinkade O.N., Olfert M.D. Geographical Prevalence of Polycystic Ovary Syndrome as Determined by Region and Race/Ethnicity. Int. J. Environ. Res. Public Health. 2018; 15(11): 2589. DOI: 10.3390/ijerph15112589

10. Knochenhauer E.S., Key T.J., Kahsar-Miller M., Waggoner W., Boots L.R., Azziz R. Prevalence of the polycystic ovary syndrome in unselected black and white women of the southeastern United States: a prospective study. J. Clin. Endocrinol. Metab. 1998; 83(9): 307882. DOI: 10.1210/jcem.83.9.5090

11. Kataoka J., Larsson I., Björkman S., Eliasson B., Schmidt J., StenerVictorin $E$. Prevalence of polycystic ovary syndrome in women with severe obesity - Effects of a structured weight loss programme. Clin. Endocrinol. (Oxf.). 2019; 91(6): 750-8. D0I: 10.1111/cen.14098

12. Zreik R.S., Nasrallah M.P. The prevalence of endocrinopathies among Lebanese women presenting with hirsutism to an endocrine clinic. J. Med. Liban. 2014; 62(1): 27-32. DOI: 10.12816/0002624

13. Norman R.J., Dewailly D., Legro R.S., Hickey T.E. Polycystic ovary syndrome. Lancet. 2007; 370(9588): 685-97. D0I: 10.1016/S01406736(07)61345-2

14. Diamanti-Kandarakis E., Dunaif A. Insulin resistance and the polycystic ovary syndrome revisited: an update on mechanisms and implications. Endocr. Rev. 2012; 33(6): 981-1030. D0I: 10.1210/er.2011-1034

15. Legro R.S., Kunselman A.R., Dunaif A. Prevalence and predictors of dyslipidemia in women with polycystic ovary syndrome. Am. J. Med. 2001; 111(8): 607-13. D0I: 10.1016/s0002-9343(01)00948-2

16. Teede H.J., Misso M.L., Boyle J.A., Garad R.M., McAllister V., Downes $L$. et al. Translation and implementation of the Australianled PCOS guideline: clinical summary and translation resources from фенотипа СПКЯ. Принимая во внимание повышенные риски нарушений углеводного обмена, сердечно-сосудистых заболеваний и депрессивных состояний при СПКЯ (особенно у женщин с ожирением), ведение пациенток следует осуществлять на междисциплинарной основе.

Своевременная диагностика СПКЯ является необходимым условием раннего начала терапии и, соответственно, профилактики его осложнений.

the International Evidence-based Guideline for the Assessment and Management of Polycystic Ovary Syndrome. Med. J. Aust. 2018; 209(7): S3-8. DOI: 10.5694/mja18.00656

17. Qin J.Z., Pang L.H., Li M.J., Fan X.J., Huang R.D., Chen H.Y. Obstetric complications in women with polycystic ovary syndrome: a systematic review and meta-analysis. Reprod. Biol. Endocrinol. 2013; 11: 56. DOI: 10.1186/1477-7827-11-56

18. Лесной С.К. Частичная резекция яичников при олигоменорее и аменорее. Акушерство и гинекология. 1928; 7: 16-9. [Lesnoi S.K. Partial ovarian resection in oligomenorrhea and amenorrhea. Obstetrics and Gynecology. 1928, 7: 16-9 (in Russian)]

19. Stein I.F., Leventhal M.L. Amenorrhea associated with bilatera polycystic ovaries. Am. J. Obstet. Gynecol. 1935; 29 (18): 1-191.

20. Nehir Aytan A., Bastu E., Demiral I., Bulut H., Dogan M., Buyru F. Relationship between hyperandrogenism, obesity, inflammation and polycystic ovary syndrome. Gynecol. Endocrinol. 2016; 32(9): 709_ 13. DOI: $10.3109 / 09513590.2016 .1155208$

21. Zore T., Lizneva D., Brakta S., Walker W., Suturina L., Azziz R. Minima Difference in Phenotype Between Adolescents and Young Adults With Polycystic Ovary Syndrome. Fertil. Steril. 2019; 111(2): 389-96. DOI: 10.1016/j.fertnstert.2018.10.020

22. Rojas J., Chávez M., Olivar L., Rojas M., Morillo J., Mejías J. et al. Polycystic ovary syndrome, insulin resistance, and obesity: navigating the pathophysiologic labyrinth. Int. J. Reprod. Med. 2014; 2014: 719050. DOI: 10.1155/2014/719050

23. Azziz R. Polycystic Ovary Syndrome. Obstet. Gynecol. 2018; 132(2): 321-36. DOI: 10.1097/AOG.0000000000002698

24. Carvalho L.M.L., Dos Reis F.M., Candido A.L., Nunes F.F.C., Ferreira C.N., Gomes K.B. Polycystic Ovary Syndrome as a systemic disease with multiple molecular pathways: a narrative review. Endocr. Regul. 2018; 52(4): 208-21. DOI: 10.2478/enr-2018-0026

25. Franks S., Hardy K. Androgen Action in the Ovary. Front. Endocrinol. (Lausanne). 2018; 9: 452. D0I: 10.3389/fendo.2018.00452

26. Di Segni C., Silvestrini A., Fato R., Bergamini C., Guidi F., Raimondo S. et al. Plasmatic and Intracellular Markers of Oxidative Stress in Normal Weight and Obese Patients with Polycystic Ovary Syndrome. Exp. Clin. Endocrinol. Diabetes. 2017; 125(8): 506-13. D0I: 10.1055/s-0043111241

27. Даренская М.А., Колесников С.И., Гребенкина Л.А., Данусевич И.Н., Лазарева Л.М., Наделяева Я.Г. и др. Анализ про- и антиоксидантной активности крови у женщин с различными фенотипами синдрома поликистозных яичников и бесплодием. Акушерство и гинекология. 2017; 8: 86-91. [Darenskaya M.A., Kolesnikov S.I., Grebenkina L.A., Danusevich I.N., Lazareva L.M., Nadelyaeva Ya.G. et al. Evaluation of prooxidant and antioxidant blood activity in women with various phenotypes of polycystic ovary syndrome and infertility. Obstetrics and Gynecology. 2017, 8: 86-91. (in Russian)] DOI: 10.18565/aig.2017.8.86-91

28. Sarray S., Madan S., Saleh L.R., Mahmoud N., Almawi W.Y. Validity of adiponectin-to-leptin and adiponectin-to-resistin ratios as predictors of polycystic ovary syndrome. Fertil. Steril. 2015; 104(2): 460-6. DOI: 10.1016/j.fertnstert.2015.05.007

29. Zawadzki J.K., Dunaif A. Diagnostic criteria for polycystic ovary syndrome: Towards a rational approach, polycystic ovary syndrome. In: Dunaif A., Givens J.R., Haseltine F., eds. Polycystic Ovary Syndrome. Boston: Blackwell Scientific Publications; 1992: 377-84.

30. The Rotterdam ESHRE/ASRM sponsored PCOS consensus workshop group. Revised 2003 consensus on diagnostic criteria and long-term health risks related to polycystic ovary syndrome (PCOS). Hum. Reprod. 2004; 19(1): 41-7. D0I: 10.1093/humrep/deh098

31. Carmina E., Azziz R. Diagnosis, Phenotype, and Prevalence of Polycystic Ovary Syndrome. Fertil. Steril. 2006; 86 (Suppl. 1): S7-8. DOI: 10.1016/j.fertnstert.2006.03.012 
32. Azziz R., Carmina E., Dewailly D., Diamanti-Kandarakis E., EscobarMorreale H.F., Futterweit W. et al. Positions statement: criteria for defining polycystic ovary syndrome as a predominantly hyperandrogenic syndrome: an Androgen Excess Society guideline. J. Clin. Endocrinol. Metab. 2006; 91(11): 4237-45. D0I: 10.1210/jc.2006-0178

33. Azziz R., Carmina E., Chen Z., Dunaif A., Laven J.S., Legro R.S. et al. Polycystic ovary syndrome. Nat. Rev. Dis. Primers. 2016; 2: 16057. DOI: $10.1038 /$ rdp. 2016.57

34. Panidis D., Tziomalos K., Papadakis E., Chatzis P., Kandaraki E.A., Tsourdi E.A. et al. Associations of menstrual cycle irregularities with age, obesity and phenotype in patients with polycystic ovary syndrome. Hormones (Athens). 2015; 14(3): 431-7. D0I:10.14310/ horm.2002.1593

35. Ferriman D., Gallwey J.D. Clinical assessment of body hair growth in women. J. Clin. Endocrinol. Metab. 1961; 21: 1440-7. DOI: 10.1210/ jcem-21-11-1440

36. Yildiz B.O., Bolour S., Woods K., Moore A., Azziz R. Visually scoring hirsutism. Hum. Reprod. Update. 2010; 16(1): 51-64. D0I: 10.1093/ humupd/dmp024

37. Diamanti-Kandarakis E., Kouli C.R., Bergiele A.T., Filandra F.A., Tsianateli T.C., Spina G.G. et al. A survey of the polycystic ovary syndrome in the Greek island of Lesbos: hormonal and metabolic profile. J. Clin. Endocrinol. Metab. 1999; 84(11): 4006-11. DOI: 10.1210/jcem.84.11.6148

38. Asunción M., Calvo R.M., San Millán J.L., Sancho J., Avila S., EscobarMorreale H.F. A prospective study of the prevalence of the polycystic ovary syndrome in unselected Caucasian women from Spain. J. Clin. Endocrinol. Metab. 2000; 85(7): 2434-8. DOI: 10.1210/ jcem.85.7.6682

39. Zargar A.H., Wani A.I., Masoodi S.R., Laway B.A., Bashir M.I. Salahuddin M. Epidemiologic and etiologic aspects of hirsutism in Kashmiri women in the Indian subcontinent. Fertil. Steril. 2002; 77(4): 674-8. D0I: 10.1016/s0015-0282(01)03241-1

40. Sagsoz N., Kamaci M., OrbakZ. Body Hair Scores and Total Hair Diameters in Healthy Women in the Kirikkale Region of Turkey. Yonsei Med. J. 2004; 45(3): 483-91. D0I: 10.3349/ymj.2004.45.3.483

41. Cheewadhanaraks S., Peeyananjarassri K., Choksuchat C. Clinical Diagnosis of Hirsutism in Thai Women. J. Med. Assoc. Thai. 2004; 87(5): 459-63.

42. DeUgarte C.M., Woods K.S., Bartolucci A.A., Azziz R. Degree of facia and body terminal hair growth in unselected black and white women: toward a populational definition of hirsutism. J. Clin. Endocrinol. Metab. 2006; 91(4): 1345-50. D0I: 10.1210/jc.2004-2301

43. Mehrabian F., Khani B., Kelishadi R., Ghanbari E. The prevalence of polycystic ovary syndrome in Iranian women based on different diagnostic criteria. Endokrynol. Pol. 2011; 62(3): 238-42.

44. Teede H., Deeks A., Moran L. Polycystic ovary syndrome: a complex condition with psychological, reproductive and metabolic manifestations that impacts on health across the lifespan. BMC Med. 2010; 8: 41. DOI: 10.1186/1741-7015-8-41

45. Zhao X., Ni R., Li L., Mo Y., Huang J., Huang M. et al. Defining hirsutism in Chinese women: a cross-sectional study. Fertil. Steril. 2011; 96(3): 792-6. DOI: 10.1016/j.fertnstert.2011.06.040

46. Kim J.J., Chae S.J., Choi Y.M., Hwang S.S., Hwang K.R., Kim S.M. et al. Assessment of Hirsutism Among Korean Women: Results of a Randomly Selected Sample of Women Seeking Pre-Employment Physical Check-Up. Hum. Reprod. 2011; 26(1): 214-20. D0I: 10.1093/humrep/deq303

47. Repaci A., Gambineri A., Pasquali R. The role of low-grade inflammation in the polycystic ovary syndrome. Mol. Cell. Endocrinol. 2011; 335(1): 30-41. DOI: 10.1016/j.mce.2010.08.002

48. Escobar-Morreale H.F., Carmina E., Dewailly D., Gambineri A. Kelestimur F., Moghetti $P$. et al. Epidemiology, diagnosis and management of hirsutism: a consensus statement by the Androgen Excess and Polycystic Ovary Syndrome Society. Hum. Reprod. Update. 2012; 18(2): 146-70. D0I: 10.1093/humupd/dmr042

49. Li R., Qiao J., Yang D., Li S., Lu S., Wu X. et al. Epidemiology of hirsutism among women of reproductive age in the community: a simplified scoring system. Eur. J. Obstet. Gynecol. Reprod. Biol. 2012; 163(2): 165-9. DOI: 10.1016/j.ejogrb.2012.03.023

50. Kazemi H., Ramezani Tehrani F., Minooee S., Khalili D., Azizi F. Women self-perception of excess hair growth, as a predictor of clinical hirsutism: a population-based study. J. Endocrinol. Invest. 2015, 38(8): 923-8. DOI: 10.1007/s40618-015-0264-0

51. Harborne L., Fleming R., Lyall H., Sattar N., Norman J. Metformin or antiandrogen in the treatment of hirsutism in polycystic ovary syndrome. J. Clin. Endocrinol. Metab. 2003; 88(9): 4116-23. DOI: 10.1210/jc.2003-030424

52. Pigny P., Merlen E., Robert Y., Cortet-Rudelli C., Decanter C., Jonard S. et al. Elevated serum level of anti-mullerian hormone in patients with polycystic ovary syndrome: relationship to the ovarian follicle excess and to the follicular arrest. J. Clin. Endocrinol. Metab. 2003; 88(12): 5957-62. DOI: 10.1210/jc.2003-030727

53. Tata B., Mimouni N.E.H., Barbotin A.L., Malone S.A., Loyens A., Pigny $P$. et al. Elevated prenatal anti-Müllerian hormone reprograms the fetus and induces polycystic ovary syndrome in adulthood. Nat. Med. 2018; 24(6): 834-46. D0I: 10.1038/s41591-018-0035-5

54. Dewailly D., Andersen C.Y., Balen A., Broekmans F., Dilaver N., Fanchin $R$. et al. The physiology and clinical utility of anti-Mullerian hormone in women. Hum. Reprod. Update. 2014; 20(3): 370-85. D0I: 10.1093/humupd/dmt062

55. Abbara A., Eng P.C., Phylactou M., Clarke S.A., Hunjan T., Roberts R. et al. Anti-Müllerian hormone (AMH) in the Diagnosis of Menstrual Disturbance Due to Polycystic Ovarian Syndrome. Front. Endocrinol. (Lausanne). 2019; 10: 656. D0I: 10.3389/fendo.2019.00656

56. Чернуха Г.Е., Каприна Е.К., Найдукова А.А. Новые возможности коррекции функции репродуктивной системы при синдроме поликистозных яичников. Медицинский совет. 2015; 9: 34-8. [Chernukha G.E., Kaprina E.K., Naidukova A.A. New prospects in the management of functional reproductive disorders associated with polycystic ovary syndrome. Medical Council. 2015; 9: 34-8. (in Russian)]. DOI: 10.21518/2079-701X-2015-9-34-38

57. Pigny P., Gorisse E., Ghulam A., Robin G., Catteau-Jonard S., Duhamel A. et al. Comparative assessment of five serum antimüllerian hormone assays for the diagnosis of polycystic ovary syndrome. Fertil. Steril. 2016; 105(4): 1063-9.e3. DOI: 10.1016/j.fertnstert.2015.12.023

58. Tasali E., Van Cauter E., Ehrmann D.A. Relationships between sleep disordered breathing and glucose metabolism in polycystic ovary syndrome. J. Clin. Endocrinol. Metab. 2006; 91(1): 36-42. DOI: 10.1210/jc.2005-1084

59. Шарифулин Э.М., Лазарева Л.М., Каня О.В., Стефаненкова А.А., Белых Д.В., Сутурина Л.В. Состояние эндометрия при синдроме поликистозных яичников в репродуктивном возрасте. Acta Biomed. Sci. 2018; 3(3): 136-42. [Sharifulin E.M., Lazareva L.M., Kanya O.V., Stefanenkova A.A., Belykh D.V., Suturina L.V. Endometrial morphology in women of reproductive age with PCOS. Acta Biomedica Scientifica. 2018; 3(3): 136-42. (in Russian)]. DOI: 10.29413/ ABS.2018-3.3.21

60. Navaratnarajah R., Pillay 0.C., Hardiman P. Polycystic ovary syndrome and endometrial cancer. Semin. Reprod. Med. 2008; 26(1): 62-71. DOI: $10.1055 / \mathrm{s}-2007-992926$

61. Piltonen T.T. Polycystic ovary syndrome: Endometrial markers. Best Pract. Res. Clin. Obstet. Gynaecol. 2016; 37: 66-79. D0I: 10.1016/j. bpobgyn.2016.03.008

62. Koster M.P., de Wilde M.A., Veltman-Verhulst S.M., Houben M.L. Nikkels P.G., van Rijn B.B. et al. Placental characteristics in women with polycystic ovary syndrome. Hum. Reprod. 2015; 30(12): 282937. DOI: $10.1093 /$ humrep/dev265

63. Hart R., Doherty D.A. The potential implications of a PCOS diagnosis on a woman's long-term health using data linkage. J. Clin. Endocrinol. Metab. 2015; 100(3): 911-9. DOI: 10.1210/jc.2014-3886

64. Sayyah-Melli M., Alizadeh M., Pourafkary N., Ouladsahebmadarek E., Jafari-Shobeiri M., Abbassi J. et al. Psychosocial Factors Associated with Polycystic Ovary Syndrome: a Case Control Study. J. Caring Sci. 2015; 4(3): 225-31. D0I: 10.15171/jcs.2015.023 D 